\title{
Teaching power system analysis courses using MATPOWER
}

\begin{abstract}
One of the major constraints faced in the teaching of power system analysis courses has been non-availability of practical power system for demonstration. This paper focuses on a new and efficient method to the teaching of power system analysis courses to the upper-division undergraduate students. The intent is to present MATPOWER to students as a good instructional tool to complement the teaching of power system analysis courses in the class. This would certainly facilitate the teaching and understanding of the subject matter better. The features and the relative merits which make the package preferred to some of the commercially available software packages, in certain applications, are highlighted in the paper. With self study in mind, the paper is written to simplify the daunting task of carrying out power flow analyses especially on large power networks. One illustrative example is examined in the paper. The power flow solution by the Newton-Raphson method is demonstrated using the conventional approach prior to solving it using the MATPOWER package. The result obtained using the package is guaranteed to be accurate and reasonably fast.
\end{abstract}

Keyword: MATLAB; MATPOWER; Jacobian matrix; Power flow; Power system analysis course; Teaching 